\title{
Bearing fault detection using multi-scale fractal dimensions based on morphological covers
}

\author{
Pei-Lin Zhang ${ }^{\mathrm{a}}$, Bing Li ${ }^{\mathrm{a}}{ }^{\mathrm{b}, *}$, Shuang-Shan Mi ${ }^{\mathrm{b}}$, Ying-Tang Zhang ${ }^{\mathrm{a}}$ and Dong-Sheng Liu $^{\mathrm{b}}$ \\ ${ }^{a}$ First Department, Mechanical Engineering College, Shijiazhuang, Hebei, China \\ ${ }^{\mathrm{b}}$ Forth Department, Mechanical Engineering College, Shijiazhuang, Hebei, China
}

Received 4 November 2010

Revised 29 October 2011

\begin{abstract}
Vibration signals acquired from bearing have been found to demonstrate complicated nonlinear characteristics in literature. Fractal geometry theory has provided effective tools such as fractal dimension for characterizing the vibration signals in bearing faults detection. However, most of the natural signals are not critical self-similar fractals; the assumption of a constant fractal dimension at all scales may not be true. Motivated by this fact, this work explores the application of the multi-scale fractal dimensions (MFDs) based on morphological cover (MC) technique for bearing fault diagnosis. Vibration signals from bearing with seven different states under four operations conditions are collected to validate the presented MFDs based on MC technique. Experimental results reveal that the vibration signals acquired from bearing are not critical self-similar fractals. The MFDs can provide more discriminative information about the signals than the single global fractal dimension. Furthermore, three classifiers are employed to evaluate and compare the classification performance of the MFDs with other feature extraction methods. Experimental results demonstrate the MFDs to be a desirable approach to improve the performance of bearing fault diagnosis.
\end{abstract}

Keywords: Rolling element bearing, fault diagnosis, feature extraction, mathematical morphology, multi-scale fractal dimensions (MFDs)

\section{Introduction}

Rolling element bearings are the most widely employed mechanical components in industrial equipments. Detecting the defects of rolling element bearing at an early stage can provide an assurance for the reliability of machine sets. One of the most widely employed techniques for bearing faults detection is the vibration signal analysis. By utilizing appropriate signal analysis methods, it is feasible to detect changes in vibration signals caused by fault components, and to make decisions about the bearing health status [1-6].

Various approaches such as Fourier transform, envelope analysis, wavelet analysis and other time-frequency techniques have been applied successfully in bearing fault diagnosis problems [7-10]. However, the signals generated by defected bearings are affected by some uncertain factors such as loads, clearance, friction, stiffness due to the complexity of the construction and working condition of rolling element bearings. Vibrations signals generated by rolling element bearings have been found to demonstrate complicated nonlinear characteristics [10-12]. The traditional signal analysis methods based on the linear system failed to represent the non-linear feature in the

*Corresponding author: Bing Li, Forth department, Mechanical Engineering College, No.97, He ping west Road, Shijiazhuang, Hebei 050003, China. Tel.: +86 31187994442 809; Fax: +86 31187994441 ; E-mail: rommandy@163.com. 
bearing fault signals. Fortunately, the non-linear dynamic theories have provided effective methods for recognizing and predicting the complex non-linear dynamic behaviors. One of the most widely used parameter is the fractal dimension, which can quantitatively characterize the non-linear behavior of vibration signal and has been broadly applied in the field of fault diagnosis [1,13-17].

There are several methods available to estimate the fractal dimension of fractals, such as the box-counting dimension, correlation dimension, information dimension and etc. Due to its simplicity and effectiveness, boxcounting technique has been widely used in various application areas [18-21]. However, researchers have pointed out that this popular approach caused under-counting at a smaller scale and over-counting at a larger scale due to its intrinsically regular partition grids scheme. Thus it produced a decreased estimation of fractal dimensions [22,23]. Therefore, a more robust scheme should be adopted to perform a more accurate estimation of the fractal dimensions.

In order to overcome the limitations of the box-counting method, a novel fractal dimension estimation technique based on mathematical morphology was proposed by Maragos [22]. The morphological erosion and dilation operations were used to create covers over a signal's graph at multiple scales for measuring the fractal dimension. This morphological covering approach unifies and extends the theoretical aspects and digital implementations of several other covering methods. It also has some computational advantages due to its computational complexity is linear with respect to both the signal's length and the maximum scale.

Moreover, for real-world signals with some fractal structure, the assumption of a constant fractal dimension at all scales may not be true. Eventually, a single non-integer number $d$ is not enough to represent all complexity of an object [24,25]. Therefore, the multi-scale fractal dimensions (MFDs), which can provide more information about the signals than the single global fractal dimension, were calculated based on the above mentioned mathematical morphological covering approach. However, there is no studies has been done on utilizing the MFDs to characterize the chaotic features of vibration signals from rolling element bearings in literature. Therefore, in this work, this newly developed multi-scale fractal dimensions (MFDs) estimation technique was applied to describe the chaotic characteristics of vibration signals from rolling element bearings with various states.

The remainders of this paper are organized as follows: Section 2 gives a brief review on the basic theories about fractals and mathematical morphology. The computation of the multi-scale fractal dimensions using morphological covers was detailed in Section 3. In Section 4, the presented MFDs estimation scheme was applied to characterize the chaotic features of vibration signals acquired from rolling element bearings. Three classifiers were utilized to evaluate the performances of the mathematical morphology based MFDs. And the conclusions of this investigation were summarized in Section 5.

\section{Brief reviews of fractal dimension and mathematic morphology theory}

\subsection{Preliminaries on fractal dimension}

Fractals are mathematical sets with a high degree of geometrical complexity that can model many natural phenomena, such as clouds, mountains, trees, and coastlines. The fractal dimension is an important parameter of fractals that describes information about their geometrical structure at multiple scales. There are several methods available to estimate the fractal dimension of fractals, such as the box-counting dimension, correlation dimension, information dimension and etc.

Due to its simplicity and effectiveness, box-counting technique has been widely used in various application areas and some improved box-counting methods were proposed, such as differential box-counting (DBC) and relative differential box-counting (RDBC). However, researchers have pointed out that this popular approach caused undercounting at a smaller scale and over-counting at a larger scale due to its intrinsically regular partition grids scheme and thus produced a decreased estimation of fractal dimensions [23]. Therefore, a more robust scheme should be adopted to perform a more accurate estimation of the fractal dimensions. 


\subsection{Basic theories on mathematical morphology}

Mathematical morphology was originally put forward as an image processing methodology by Serra [26]. And later on, it was used for one-dimensional signal processing by Magaros and Schafer [27,28]. The basic concept of morphological signal processing is to modify the shape of a signal, equivalently considered as a set, by transforming it through its interaction with another object, called the structuring element (SE). The basic operators of mathematical morphology include dilation and erosion.

Let $f(n)$ be the discrete one dimension signal over a domain $F=\{0,1,2, \cdots, N-1\}$ and let $g(m)$ be the structure element over a domain $G=\{0,1,2, \cdots M-1\}$ the dilation and erosion operators are defined as

$$
\begin{aligned}
& (f \oplus g)(n)=\max _{m \in G}\{f(n-m)+g(m)\} \\
& (f \Theta g)(n)=\min _{m \in G}\{f(n+m)-g(m)\}
\end{aligned}
$$

Where $\oplus$ represented the operator of dilation and $\Theta$ represented the operator of erosion. More details about the morphological operations and the structure elements can be found in [27].

\section{Multi-scale fractal dimensions calculated via morphological covers}

\subsection{Fractal dimension estimated by morphological covers}

Derived from the Minkowski-Bouligand Dimension, the mathematical morphology based fractal dimension replaces the Minkowski cover with the morphological cover created by morphological operations. Maragos has proven this replacement is valid and the quadratic complexity of computing the covers at all scales can be reduced to linear [22].

Let $r\left(r=1,2, \cdots, r_{\max }\right)$ to be the scale, $g$ to be a unit structure element, the structure element used at scale $r$ can be defined as:

$$
r g=\underbrace{g \oplus g \oplus \cdots \oplus g}_{r-1 \text { times }}
$$

Then the dilation and erosion of the signal $f(n)$ at the scale $r$ can be obtained:

$$
\begin{aligned}
& f \oplus r g(n)=\underbrace{f \oplus g \oplus g \oplus \cdots \oplus g}_{r-1 \text { times }}(n) \\
& f \Theta r g(n)=\underbrace{f \Theta g \Theta g \Theta \cdots \Theta g}_{r-1 \text { times }}(n)
\end{aligned}
$$

So the morphological cover $A_{g}(r)$ at scale $r$ can be defined as:

$$
A_{g}(r)=\sum_{n=1}^{N}(f \oplus r g-f \Theta r g)(n)
$$

Fit a straight line using least squares to the graph of $\log \left(A_{g}(r) / r^{2}\right)$ versus $\log (1 / r)$, the slope of this line gives us an approximate estimation of the fractal dimension $D_{M}$ of $f(n)$.

The fractal dimension $D_{M}$ of the graph of the signal $f(n)$ resulting from the morphological covering method has the following two attractive properties as described in [22]: (1) if the signal $f(n)$ is shifted, $D_{M}$ will not be changed; (2) if the structure element is a flat one, means the amplitude of the structure element is 0 , then $D_{M}$ remains invariant with respect to any affine scaling of the amplitude of $f(n)$ and/or shifting of its argument.

The morphological covering and the box counting method give identical fractal dimension for continuous time signals $f$. However, in the discrete case they correspond to different algorithms with different performances. In the 

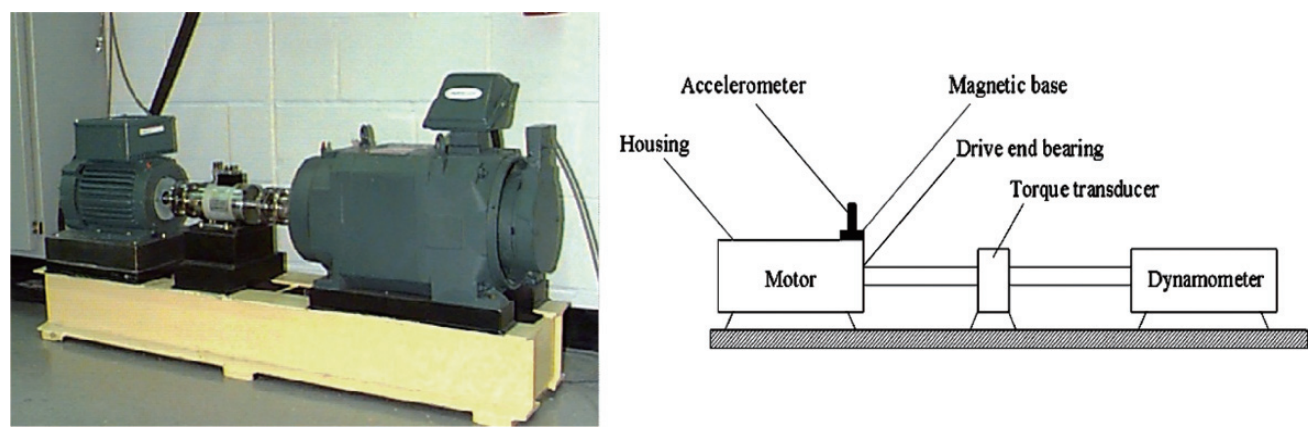

Fig. 1. Experiment system and its schematic.

discrete case, it is because of the two properties above that the morphological covering method is more robust than the box counting method. The latter is affected by arbitrary shifts of the argument off, by adding constant offsets to $f$, and (more seriously) by scaling its amplitude range, because all these affect the number of grid boxes intersected by the graph off. In addition, the box counting dimension greatly depends on the relationship between the grid spacing and the dynamic range of $f$. However, the morphological covering method using covers with one-dimensional functions $g$ can become completely independent from affine scaling of the signal's range if we choose the amplitude of the structure elements to be 0 , means the flat structure elements [22]. Throughout this paper, we have used the flat structure elements.

\subsection{Multi-scale fractal dimensions}

For real-world signals with some fractal structure, the assumption of a constant fractal dimension at all scales may not be true [24]. Different with true fractals, signals from nature are not self-similar. They may look different whether we change the visualization scale and, consequently, their Fractal Dimension is also dependent on the used scale. Eventually, a single non-integer number $d$ is not enough to represent all complexity of a signal [25]. In order to solve this deficiency in the characterization of object by complexity, the Multi-Scale Fractal Dimensions was developed.

Different from the global fractal dimension, which is computed by estimated the slope of the log-log curve, this new scheme compute the local fractal dimensions along the scales. For each scale $r$, we estimate the local fractal dimension by calculating the slope of a line segment fitted via least-squares to $\log \left(A_{g}(r) / r^{2}\right)$ versus $\log (1 / r)$ over a short moving window $(r, r+1, \cdots, r+w-1)$ of $w$ scales, where $w$ represents the length of the window. In this way, the MFDs can represent a signal by a curve along the scale, which can provide more discriminative information about the signals.

\section{Application results to bearing fault detection}

\subsection{Bearing datasets}

The vibration datasets used in this paper were acquired from the Bearing Data Center supported by the Case Western Reserve University. The detailed description about the test rig can be found in [29]. The bearing data of CWRU has been validated in many researches and has become a standard data set of the roller bearings [13,17].

The ball bearings are installed in a motor driven mechanical system, as shown in Fig. 1. A 2 hp, three-phase induction motor is connected to a dynamometer and a torque sensor by a self-aligning coupling. The dynamometer is controlled so that desired torque load levels can be achieved.

The bearing used in the experiments is the type of SKF 6205, deep groove ball bearing. Single point faults were introduced to the test bearings using electro-discharge machining. Bearings are tested under four different loads $(0,1,2$ and $3 \mathrm{hp})$. Seven different bearing states including normal states and six fault states (rolling element slight 
Table 1

Bearing dataset description

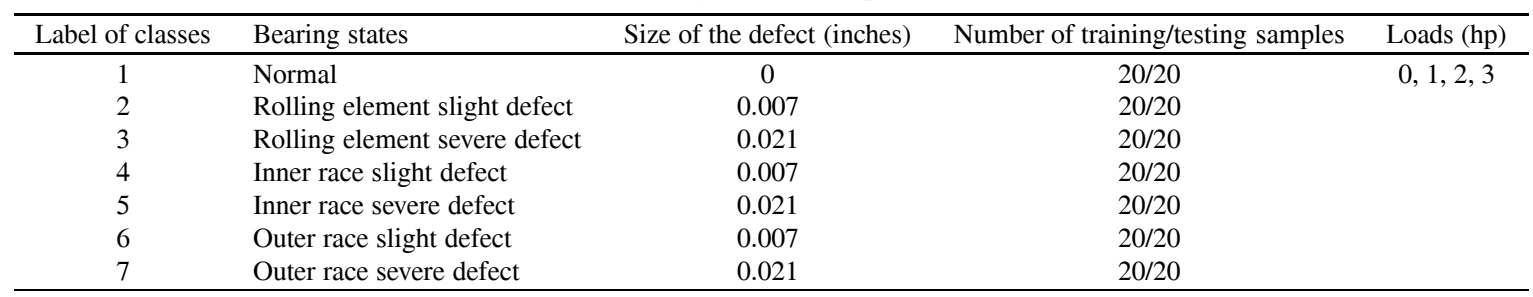
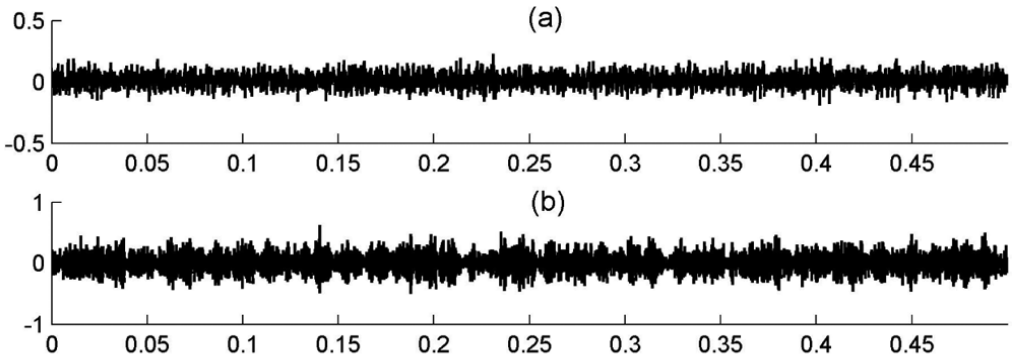

(c)
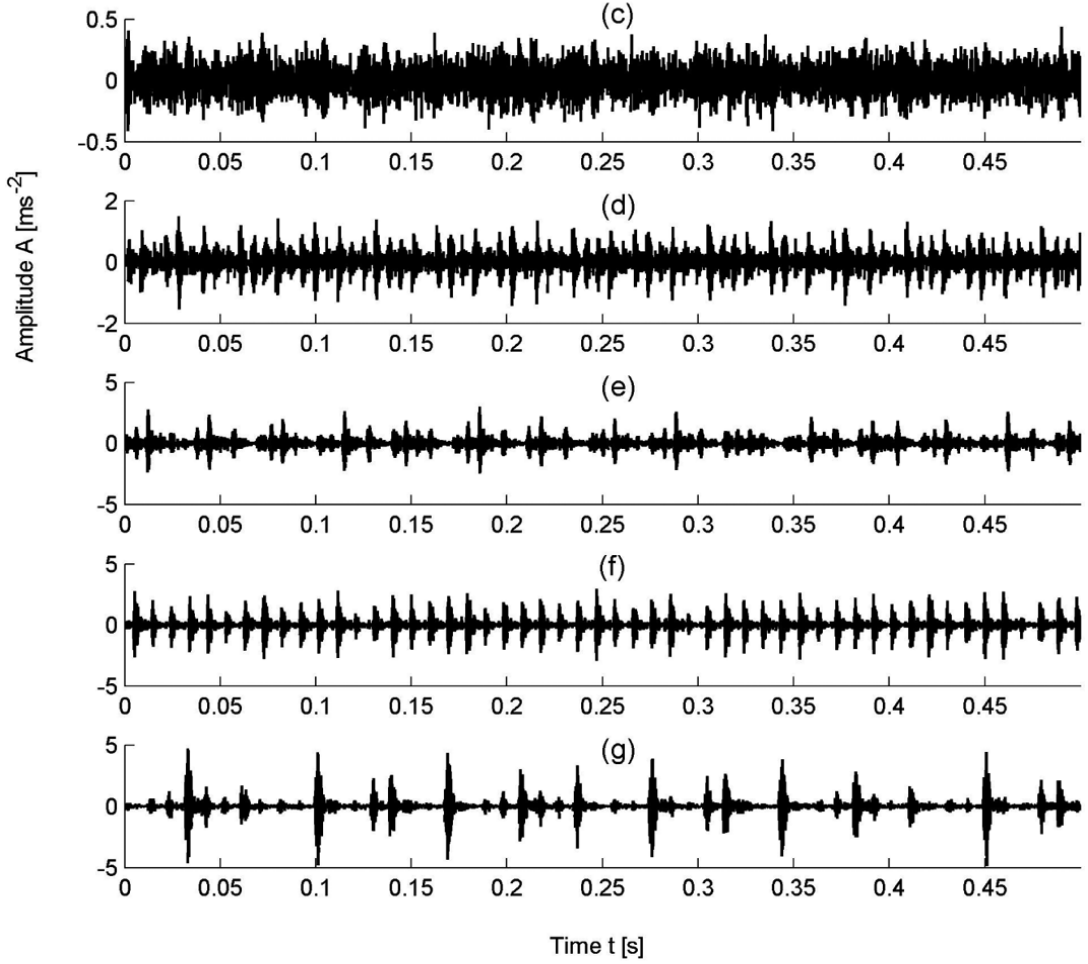

Fig. 2. Waveform of vibration signals from bearing under seven states (a)-(g) correspond to seven states (1-7) in Table 1 .

defect, rolling element severe defect, inner race slight defect£<inner race severe defect, outer race slight defect and outer race severe defect) were studied in this work. The detail description of the bearing dataset was summarized in Table 1.

Vibration data was collected using accelerometers, which were attached to the housing with magnetic bases. The sample frequency is $12000 \mathrm{~Hz}$. Forty samples containing 6000 points are collected for each bearing state under each load condition. It means that under every load condition, 280 samples were collected. Figure 2 has demonstrated the waveform of vibration signals from seven bearing states with load $3 \mathrm{hp}$. 


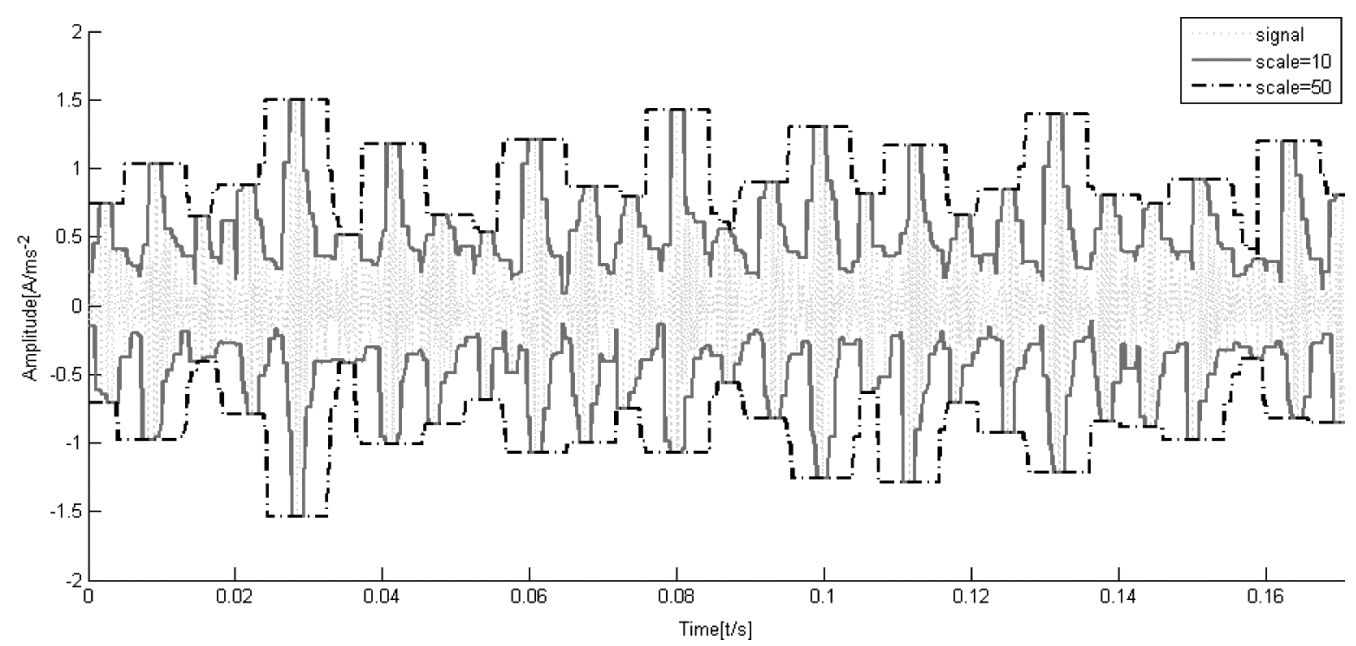

Fig. 3. Signal from outer race severe defect and its dilation and erosion by $r g$ at scale 10 and 50 (the solid line corresponding to scale 10 , the dashed line corresponding to scale 50).
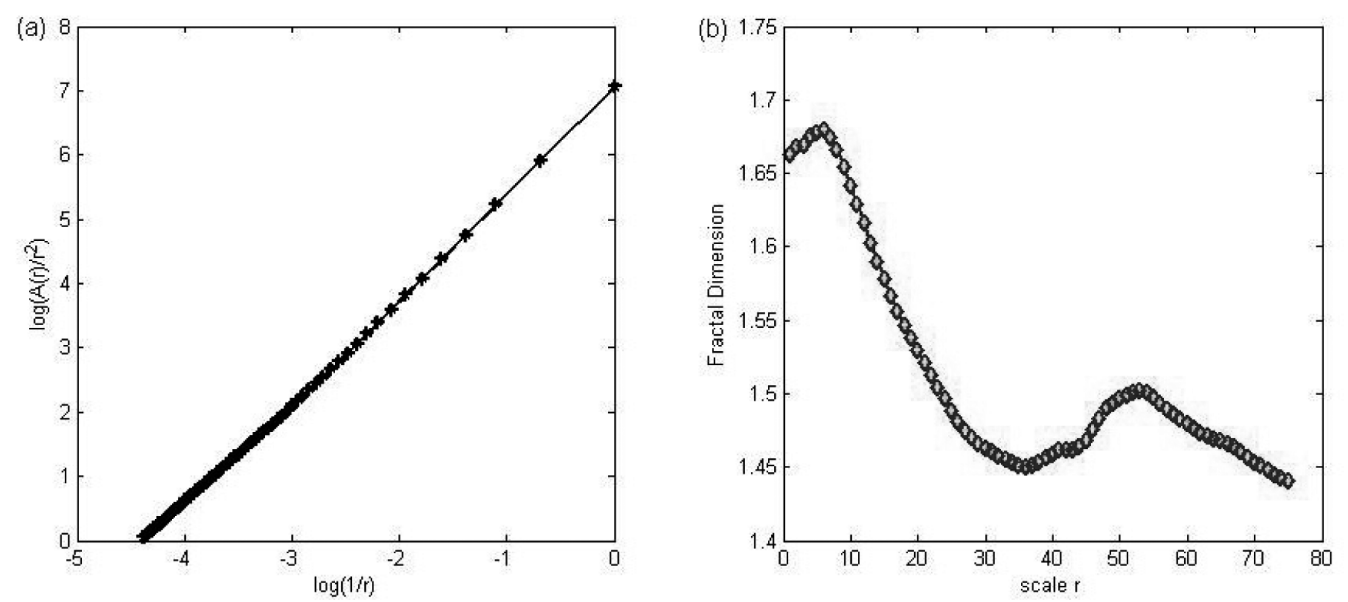

Fig. 4. (a) log-log curve of $A_{g}(r) / r^{2}$ versus $1 / r$; (b) the multi-scale fractal dimensions derived of (a).

\subsection{Multi-scale fractal dimensions of the vibration signals from bearing}

In this section, the MFDs were used to characterize the nonlinear feature of vibration signals from rolling element bearings under seven states.

The maximum scale $r_{\max }$ and the length of the moving window $w$ are two important parameters which should be selected appropriately. Unfortunately, there is no criterion has been presented for these selections. In this paper, the maximum scale $r_{\max }$ was set to be 80 and the length of the moving windows was set to be 6 based on a series of tests. More sophisticated techniques to determine these two parameters should be further studied in future works. In this way, for each signal, we can obtain 75 fractal parameters instead of only one global fractal dimension.

Take the signal acquired from bearing with outer race severe defect as an example to illustrate the calculation of the MFDs based on mathematical morphology (only 2048 points were displayed for more clear demonstration), as shown in Fig. 3. The dilation and the erosion of the original signal using flat structure elements with different scales $r$ (scale 10 and 50) were plotted in the Fig. 3. The solid lines denote the results at scale 10 and the dashed lines denote the results at scale 50. The upper lines denote the dilation results and the lower lines denote the erosion results of the signal. And the area between the upper line and lower line is the cover area to be calculated as $A_{g}(r)$. 


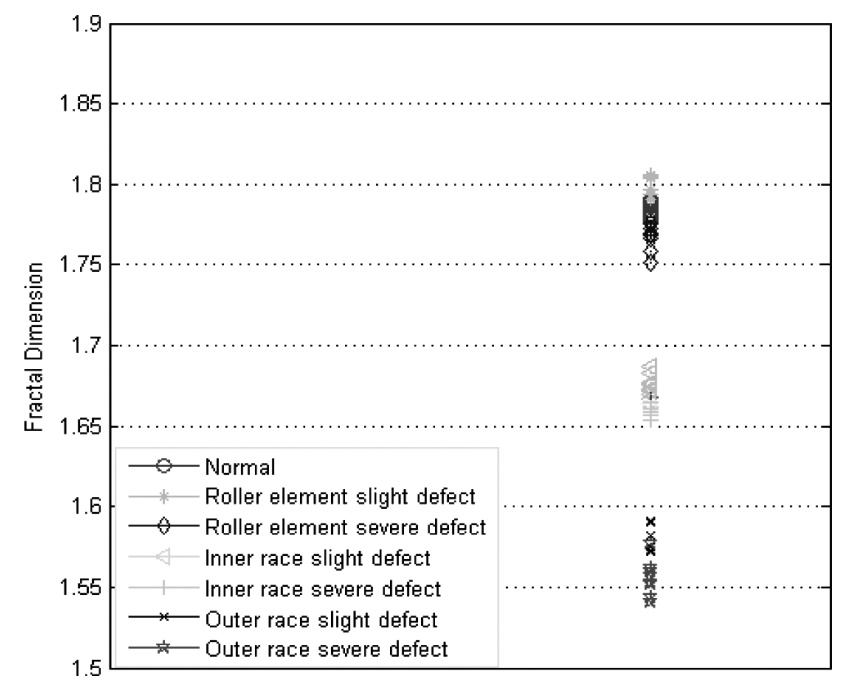

Fig. 5. Single global fractal dimensions of seven bearing states.

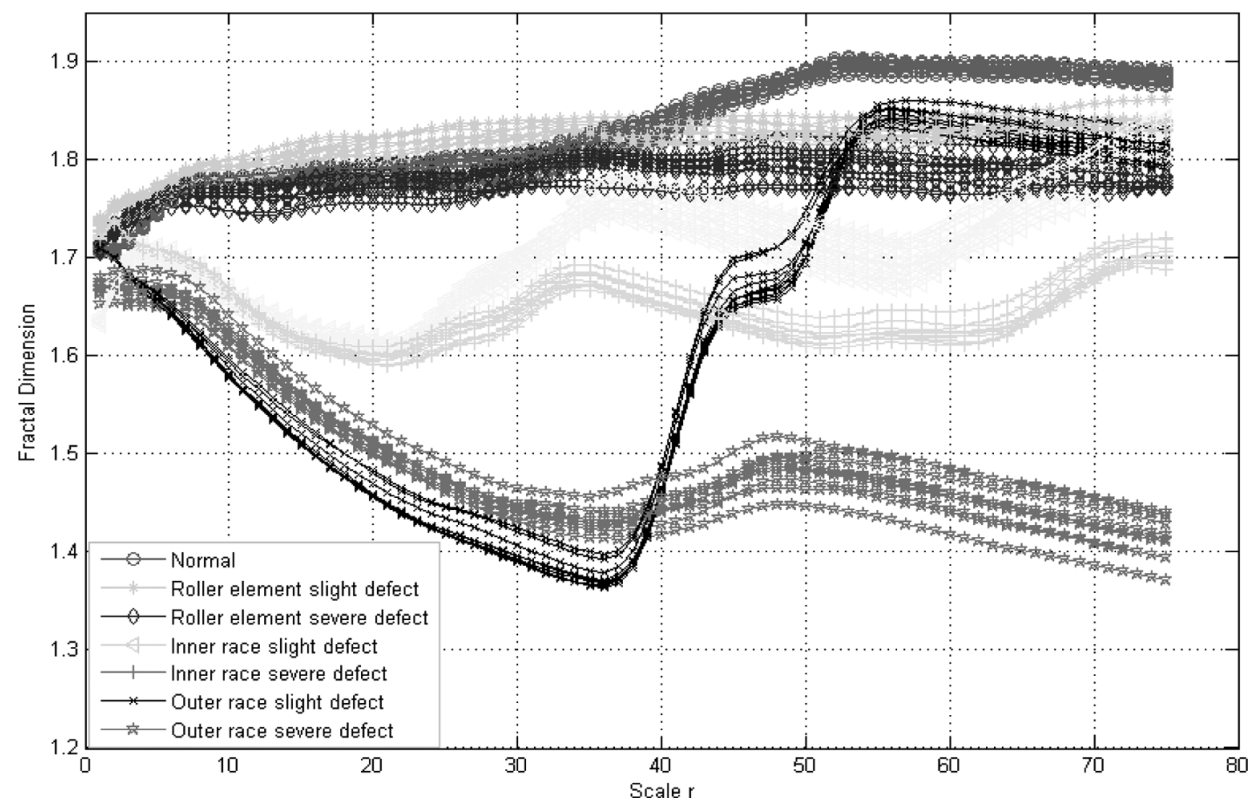

Fig. 6. Multi-scale fractal dimensions of seven bearing states.

The multi-scale fractal dimensions (MFDs) of the signal displayed in Fig. 3 calculated by the presented scheme were given in Fig. 4. The log-log curve of $A_{g}(r) / r^{2}$ versus $1 / r$ were plotted in Fig. 4(a) and the corresponding multi-scale fractal dimensions were illustrated in Fig. 4(b).

It can be observed that the signal of defected rolling element bearing demonstrates varied dimensions at different scales. It confirms our understanding that the signal from real world may contain different fractal structures at different scales.

The single global fractal dimension $F D_{M M}$ of seven bearing states were also calculated by the morphological covers and shown in Fig. 5. Five samples of each bearing state have been displayed in Fig. 5. It can be found that the $F D_{M M}$ can not distinguish the seven bearing states very well.

Figure 6 demonstrates the MFDs of vibration signals from seven states of rolling element bearing. We can obviously find that the fractal dimensions of vibration signals from bearing with seven states are varied along with 
Table 2

Performances of Wavelet-Statistic, $F D_{B O X}, F D_{M M}$ and $M F D$ s using the three classifiers on four bearing datasets

\begin{tabular}{|c|c|c|c|c|c|}
\hline \multicolumn{2}{|c|}{ Operating condition } & \multirow[t]{2}{*}{ Methods } & \multicolumn{3}{|c|}{ Classification rates } \\
\hline Rotor speed (rpm) & Load (hp) & & KNNC & NBC & LS-SVM \\
\hline 1797 & 0 & $\begin{array}{c}\text { Wavelet-Statistic } \\
\qquad \begin{array}{c}B D_{B O X} \\
F D_{M M} \\
M F D s\end{array}\end{array}$ & $\begin{array}{l}0.9714 \\
0.7943 \\
0.8327 \\
1.0000\end{array}$ & $\begin{array}{l}0.9829 \\
0.6171 \\
0.7306 \\
0.9943\end{array}$ & $\begin{array}{l}0.9771 \\
0.8114 \\
0.9388 \\
1.0000\end{array}$ \\
\hline 1772 & 1 & $\begin{array}{l}\text { Wavelet-Statistic } \\
\qquad \begin{array}{c}F D_{B O X} \\
F D_{M M} \\
M F D s\end{array}\end{array}$ & $\begin{array}{l}0.9543 \\
0.8057 \\
0.8980 \\
1.0000\end{array}$ & $\begin{array}{l}0.8971 \\
0.6914 \\
0.7918 \\
0.9657\end{array}$ & $\begin{array}{l}0.9624 \\
0.8343 \\
0.8898 \\
1.0000\end{array}$ \\
\hline 1750 & 2 & $\begin{array}{l}\text { Wavelet-Statistic } \\
\qquad \begin{array}{c}F D_{B O X} \\
F D_{M M} \\
M F D s\end{array}\end{array}$ & $\begin{array}{l}0.8804 \\
0.7914 \\
0.9306 \\
1.0000\end{array}$ & $\begin{array}{l}0.9714 \\
0.7400 \\
0.9184 \\
0.9943\end{array}$ & $\begin{array}{l}1.0000 \\
0.7800 \\
0.8857 \\
1.0000\end{array}$ \\
\hline 1730 & 3 & $\begin{array}{c}\text { Wavelet-Statistic } \\
\qquad F D_{B O X} \\
F D_{M M} \\
M F D s \\
\end{array}$ & $\begin{array}{l}0.9371 \\
0.8171 \\
0.9469 \\
\mathbf{1 . 0 0 0 0} \\
\end{array}$ & $\begin{array}{l}0.8971 \\
0.7429 \\
0.8857 \\
\mathbf{1 . 0 0 0 0} \\
\end{array}$ & $\begin{array}{l}0.9943 \\
0.8114 \\
0.9469 \\
\mathbf{1 . 0 0 0 0} \\
\end{array}$ \\
\hline
\end{tabular}

the scale. It also can be observed that in some scale the seven states overlapped with each other, but they can be distinguished very clearly at other scales. So the multi-scale fractal dimensions can provide more discriminative information about the signals than the single global fractal dimension $F D_{M M}$.

\subsection{Classification performance of MFDs}

In this section, the capacity of the MFDs to distinguish seven bearing states was evaluated. We conduct the experiments on four bearing datasets, mean bearing datasets under four different operation conditions, as shown in Table 1.

Three popular classifiers mean the nearest neighbor classifier (KNNC) [30], nearest mean classifier (NMC) [31] and least-square support vector machine (LS-SVM) [32] were employed to classify the seven states of rolling element bearings by utilizing the MFDs as input feature vector. The KNNC and NMC were implemented by utilizing the Matlab Toolbox for Pattern Recognition (PRTools 4.1) [33]. The LS-SVM was implemented by the LS-SVMlab1.5, which can be downloaded from [34]. The parameters of the LS-SVM were optimized by cross-validation method.

As mentioned in section 4.1, for every operation state, 40 samples were collected. The dataset was segmented to two parts, 140 samples (20 samples of each state) were employed as the training dataset and the other 140 samples were used as testing dataset. We did this segmentation randomly for 20 times to get a more accurate evaluation results.

For comparison, the single global fractal dimension (denoted as $F D_{M M}$ ) and box-counting fractal dimension (denoted as $F D_{B O X}$ ) were also computed and used as input vectors for classification. Some traditional feature extraction approaches should be employed to compare with the proposed method. It is not realistic to compare all the feature extraction methods in literatures. The statistical parameters and the wavelet transform are the most popular used techniques for bearing fault diagnosis over the past few years [35-41]. So we just incorporated the statistical analysis and wavelet transform to generate another feature subset for comparison. The details about these two techniques can be found in the mentioned studies. In this work, the ' $\mathrm{db} 5$ ' wavelet was utilized to decompose the original signal to series of coefficients. The decomposition level was set to be 4 . Then five coefficients including one approximation coefficient and four detail coefficients can be obtained for each sample. Ten static parameters means the mean, standard derivation, root mean square, maximum, skewness, kurtosis, crest factor, impulse factor, shape factor and clearance factor were calculated for each coefficient. Therefore, we can obtain ten features for every coefficient. In this way, fifty features can be calculated for each sample based on the statistical analysis and wavelet transform. 
Table 3

Performances of Wavelet-Statistic, $F D_{B O X}, F D_{M M}$ and $M F D$ s using the three classifiers on four 'leaving one operation condition out' bearing datasets

\begin{tabular}{|c|c|c|c|c|c|}
\hline \multicolumn{2}{|c|}{ The leaving out operating condition } & \multirow[t]{2}{*}{ Methods } & \multicolumn{3}{|c|}{ Classification rates } \\
\hline Rotor speed (rpm) & Load (hp) & & KNNC & NBC & SVC \\
\hline \multirow[t]{4}{*}{1797} & 0 & Wavelet-Statistic & 0.8286 & 0.7571 & 0.8857 \\
\hline & & $F D_{B O X}$ & 0.6571 & 0.7286 & 0.6857 \\
\hline & & $F D_{M M}$ & 0.7571 & 0.7429 & 0.7714 \\
\hline & & $M F D s$ & 0.8429 & 0.9143 & 0.9143 \\
\hline \multirow[t]{4}{*}{1772} & 1 & Wavelet-Statistic & 0.8286 & 0.8286 & 0.8429 \\
\hline & & $F D_{B O X}$ & 0.6571 & 0.6571 & 0.7571 \\
\hline & & $F D_{M M}$ & 0.7571 & 0.8 & 0.8429 \\
\hline & & $M F D s$ & 0.9 & 0.9714 & 0.9571 \\
\hline \multirow[t]{4}{*}{1750} & 2 & Wavelet-Statistic & 0.9 & 0.9143 & 0.8857 \\
\hline & & $F D_{B O X}$ & 0.7429 & 0.8143 & 0.7857 \\
\hline & & $F D_{M M}$ & 0.8143 & 0.8 & 0.8143 \\
\hline & & $M F D s$ & 0.9 & 0.9571 & 0.9857 \\
\hline \multirow[t]{4}{*}{1730} & 3 & Wavelet-Statistic & 0.8857 & 0.9143 & 0.9 \\
\hline & & $F D_{B O X}$ & 0.7571 & 0.7286 & 0.7286 \\
\hline & & $F D_{M M}$ & 0.8143 & 0.8286 & 0.8857 \\
\hline & & $M F D s$ & 0.9857 & 0.9429 & \\
\hline
\end{tabular}

The performances of $M F D s, F D_{M M}, F D_{B O X}$ and Wavelet-Statistic features using the three classifiers on four bearing datasets were given in Table 2. An observation from Table 2 is that the MFDs are always outperforming the $F D_{M M}$ and $F D_{B O X}$ by using different classifiers on four bearing datasets. It also can be found that the Wavelet-Statistic also outperforms the $F D_{M M}$ and $F D_{B O X}$, but it is still inferior to the MFDs.

Furthermore, we have also conducted another experiment to verify the generalizing capacity of MFDs. In this experiment, we take the 'leaving one operation condition out' strategy to test and compare the generalizing capacity of the above-mentioned four different feature subsets. Firstly, we select out one operation condition from the four operation conditions. Secondly, all the samples of the selected operation condition are used as the testing dataset and all the samples of other three operation conditions are used as the training dataset. For example, if we leaving the first operation condition out, all the samples in the first condition will be used as the testing dataset. And all the samples from other three operation conditions will be used as training dataset. In this way, we can testify the generalizing capacity of the four feature subsets on different operation conditions. Table 3 shows the classification performances of the four feature subsets in our 'leaving one operation condition out' experiments.

It can be found in Table 3 that the classification performance of MFDs is also very satisfactory when the operation condition varied. It indicates that the excellent generalized capacity of the MFDs in bearing fault diagnosis. It also can be observed that the MFDs demonstrate the best classification performance among the four feature subsets in our 'leaving one operation condition out' experiments. It is due to the fact that the MFDs contain more information about the signals than a global estimation of fractal dimension. It is desirable to utilize MFDs to extract more informative features for fault diagnosis of bearing with different defect types under various loads.

\section{Conclusion}

The multi-scale fractal dimensions (MFDs) estimated by morphological covering technique has been introduced to detect the defects of rolling element bearings in this work. Application results have revealed that the vibration signals acquired from bearing with seven states demonstrate different fractal structures when the visualization scales were changed. The multi-scale fractal dimensions can provide more discriminative information about the signals than the single global fractal dimension.

The performances of wavelet-statistical features, single fractal dimension estimated by MM, the box-counting fractal dimension and the presented MFDs were evaluated on four different data sets by using three classifiers. Results showed that the MFDs outperform the other three techniques very clearly in detecting bearing defects under varied operating conditions. We also conduct an experiment using 'leaving one operation condition out' strategy to 
verify the generalizing capacity of the presented MFDs. Experimental results have demonstrated that the generalizing capacity of the MFDs is also very satisfactory in bearing fault diagnosis.

This research has demonstrated clearly that the MFDs based on mathematical morphology have great potential to be an effective and efficient tool for the diagnosis of rolling element bearings and can be extended to diagnose other rotating machineries.

\section{Acknowledgments}

This research is supported by the National Natural Science Foundation of China (No. 50705097) and Natural Science Foundation of He-bei Province (No. E2007001048).

\section{References}

[1] C.H. Chen, R.J. Shyu and C.K. Ma, A new fault diagnosis method of rotating machinery, Shock and Vibration 6 (2008), 585-598.

[2] J. Cheng, D. Yu, J. Tang and Y. Yang, Application of SVM and SVD technique based on EMD to the fault diagnosis of the rotating machinery, Shock and Vibration 1 (2009), 89-98.

[3] R.G.T. De Almeida, S.A. Da Silva Vicente and L.R. Padovese, New technique for evaluation of global vibration levels in rolling bearings, Shock and Vibration 4-5 SPEC (2002), 225-234.

[4] C.T. Yiakopoulos and I.A. Antoniadis, Wavelet based demodulation of vibration signals generated by defects in rolling element bearings, Shock and Vibration 6 (2002), 293-306.

[5] Y.C. Choi and Y.H. Kim, Fault detection in a ball bearing system using minimum variance cepstrum, Measurement Science and Technology 5 (2007), 1433-1440.

[6] X. Wang, Y. Zi and Z. He, Multiwavelet construction via an adaptive symmetric lifting scheme and its applications for rotating machinery fault diagnosis, Measurement Science and Technology 4 (2009), 1-17.

[7] Y.D. Chen, R. Du and L.S. Qu, Fault features of large rotating machinery and diagnosis using sensor fusion, Journal of Sound and Vibration 2 (1995), 227-242.

[8] B. Liu, Selection of wavelet packet basis for rotating machinery fault diagnosis, Journal of Sound and Vibration 3-5 (2005), 567-582.

[9] Y.T. Sheen, A complex filter for vibration signal demodulation in bearing defect diagnosis, Journal of Sound and Vibration 1-2 (2004), $105-119$.

[10] Y. Yu, Y. Dejie and C. Junsheng, A roller bearing fault diagnosis method based on EMD energy entropy and ANN, Journal of Sound and Vibration 1-2 (2006), 269-277.

[11] C.L. Chen and H.T. Yau, Chaos in the Imbalance Response of a Flexible Rotor Supported by Oil Film Bearings with Non-Linear Suspension, Nonlinear Dynamics 1 (1998), 71-90.

[12] R.D. Brown, P. Addison and A.H.C. Chan, Chaos in the unbalance response of journal bearings, Nonlinear Dynamics 4 (1994), $421-432$.

[13] J. Yang, Y. Zhang and Y. Zhu, Intelligent fault diagnosis of rolling element bearing based on SVMs and fractal dimension, Mechanical Systems and Signal Processing 5 (2007), 2012-2024.

[14] C. Craig, R.D. Neilson and J. Penman, The use of correlation dimension in condition monitoring of systems with clearance, Journal of Sound and Vibration 1 (2000), 1-17.

[15] D. Logan and J. Mathew, Using the correlation dimension for vibration fault diagnosis of rolling element bearings - I. Basic concepts, Mechanical Systems and Signal Processing 3 (1996), 241-250.

[16] J.D. Jiang, J. Chen and L.S. Qu, The application of correlation dimension in gearbox condition monitoring, Journal of Sound and Vibration 4 (1999), 529-541.

[17] W.J. Wang, J. Chen, X.K. Wu and Z.T. Wu, The application of some non-linear methods in rotating machinery fault diagnosis, Mechanical Systems and Signal Processing 4 (2001), 697-705.

[18] C.H. Chen, R.J. Shyu and C.K. Ma, Rotating machinery diagnosis using wavelet packets-fractal technology and neural networks, Journal of Mechanical Science and Technology 7 (2007), 1058-1065.

[19] L.S. Liebovitch and T. Toth, A fast algorithm to determine fractal dimensions by box counting, Physics Letters A 8-9 (1989), 386-390.

[20] F. Camastra and A. Vinciarelli, Estimating the intrinsic dimension of data with a fractal-based method, IEEE Transactions on Pattern Analysis and Machine Intelligence 10 (2002), 1404-1407.

[21] T. Marwala, U. Mahola and F.V. Nelwamondo, Hidden markov models and gaussian mixture models for bearing fault detection using fractals, in: IEEE International Conference on Neural Networks - Conference Proceedings, 2006, 3237-3242.

[22] P. Maragos and F.-K. Sun, Measuring the fractal dimension of signals: morphological covers and iterative optimization, IEEE Transactions on Signal Processing 1 (1993), 108-121.

[23] Y. Xia, D. Feng and R. Zhao, Morphology-based multifractal estimation for texture segmentation, IEEE Transactions on Image Processing 3 (2006), 614-623.

[24] P. Maragos and A. Potamianos, Fractal dimensions of speech sounds: Computation and application to automatic speech recognition, Journal of the Acoustical Society of America 3 (1999), 1925-1932.

[25] A.R. Backes and O.M. Bruno, Shape classification using complex network and Multi-scale Fractal Dimension, Pattern Recognition Letters 1 (2010), 44-51. 
[26] J. Serra, Introduction to mathematical morphology, Computer Vision, Graphics, and Image Processing 3 (1986), $283-305$.

[27] P. Maragos and R.W. Schafer, Morphological filters - Part i: Their set - Theoretic analysis and relations to linear shift - invariant filters, IEEE Transactions on Acoustics, Speech, and Signal Processing 8 (1987), 1153-1169.

[28] P. Maragos and R.W. Schafer, Morphological filters - Part ii: Their relations to median, order - statistic, and stack filters, IEEE Transactions on Acoustics, Speech, and Signal Processing 8 (1987), 1170-1184.

[29] K.A. Loparo, Case Western Reserve University, http://www.eecs.cwru.edu/laboratory/bearing/download.htm (2000).

[30] P.J. Grother, G.T. Candela and J.L. Blue, Fast implementations of nearest neighbor classifiers, Pattern Recognition 3 (1997), $459-465$.

[31] C.J. Veenman and M.J.T. Reinders, The nearest subclass classifier: A compromise between the nearest mean and nearest neighbor classifier, IEEE Transactions on Pattern Analysis and Machine Intelligence 9 (2005), 1417-1429.

[32] J.A.K. Suykens and J. Vandewalle, Least squares support vector machine classifiers, Neural Processing Letters 3 (1999), $293-300$.

[33] P.J. R.P.W. Duin, P. Paclik, E. Pekalska, D. de Ridder, D.M.J. Tax and S. Verzakov, PRTools4.1, A Matlab Toolbox for Pattern Recognition, Delft University of Technology (2007).

[34] LS-SVMlab1.5, http://www.esat.kuleuven.ac.be/sista/lssvmlab/.

[35] B. Samanta and K.R. Al-Balushi, Artificial neural network based fault diagnostics of rolling element bearings using time-domain features, Mechanical Systems and Signal Processing 2 (2003), 317-328.

[36] Q. Sun, P. Chen, D. Zhang and F. Xi, Pattern recognition for automatic machinery fault diagnosis, Journal of Vibration and Acoustics, Transactions of the ASME 2 (2004), 307-316.

[37] Q. Hu, Z. He, Z. Zhang and Y. Zi, Fault diagnosis of rotating machinery based on improved wavelet package transform and SVMs ensemble, Mechanical Systems and Signal Processing 2 (2007), 688-705.

[38] Y. Lei, Z. He and Y. Zi, A new approach to intelligent fault diagnosis of rotating machinery, Expert Systems with Applications 4 (2008), 1593-1600.

[39] T. Karacay and N. Akturk, Experimental diagnostics of ball bearings using statistical and spectral methods, Tribology International 6 (2009), 836-843.

[40] P. Stepanic, I.V. Latinovic and Z. Djurovic, A new approach to detection of defects in rolling element bearings based on statistical pattern recognition, International Journal of Advanced Manufacturing Technology (2009), 1-10.

[41] Y. Lei, Z. He and Y. Zi, Application of an intelligent classification method to mechanical fault diagnosis, Expert Systems with Applications 6 (2009), 9941-9948. 

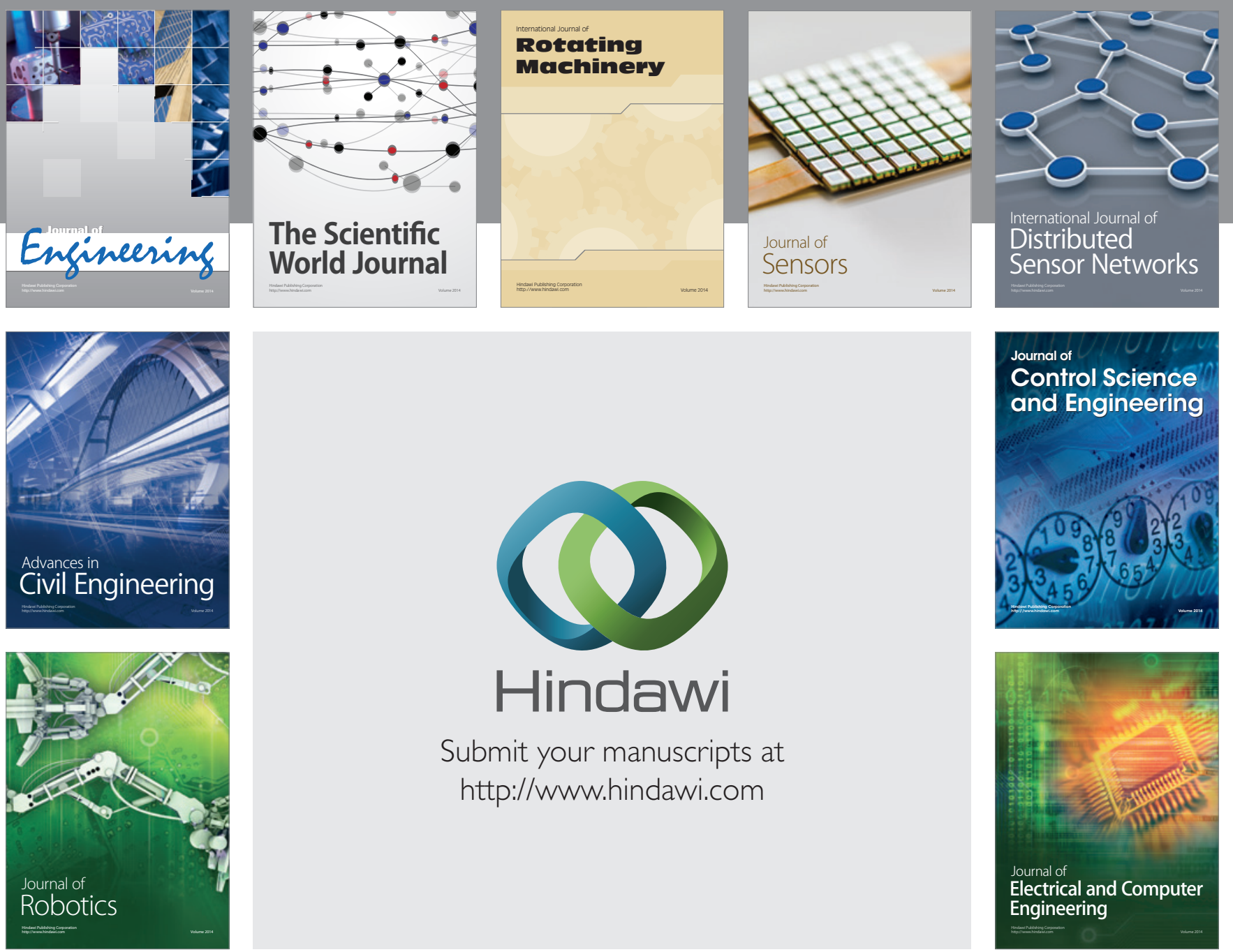

Submit your manuscripts at

http://www.hindawi.com
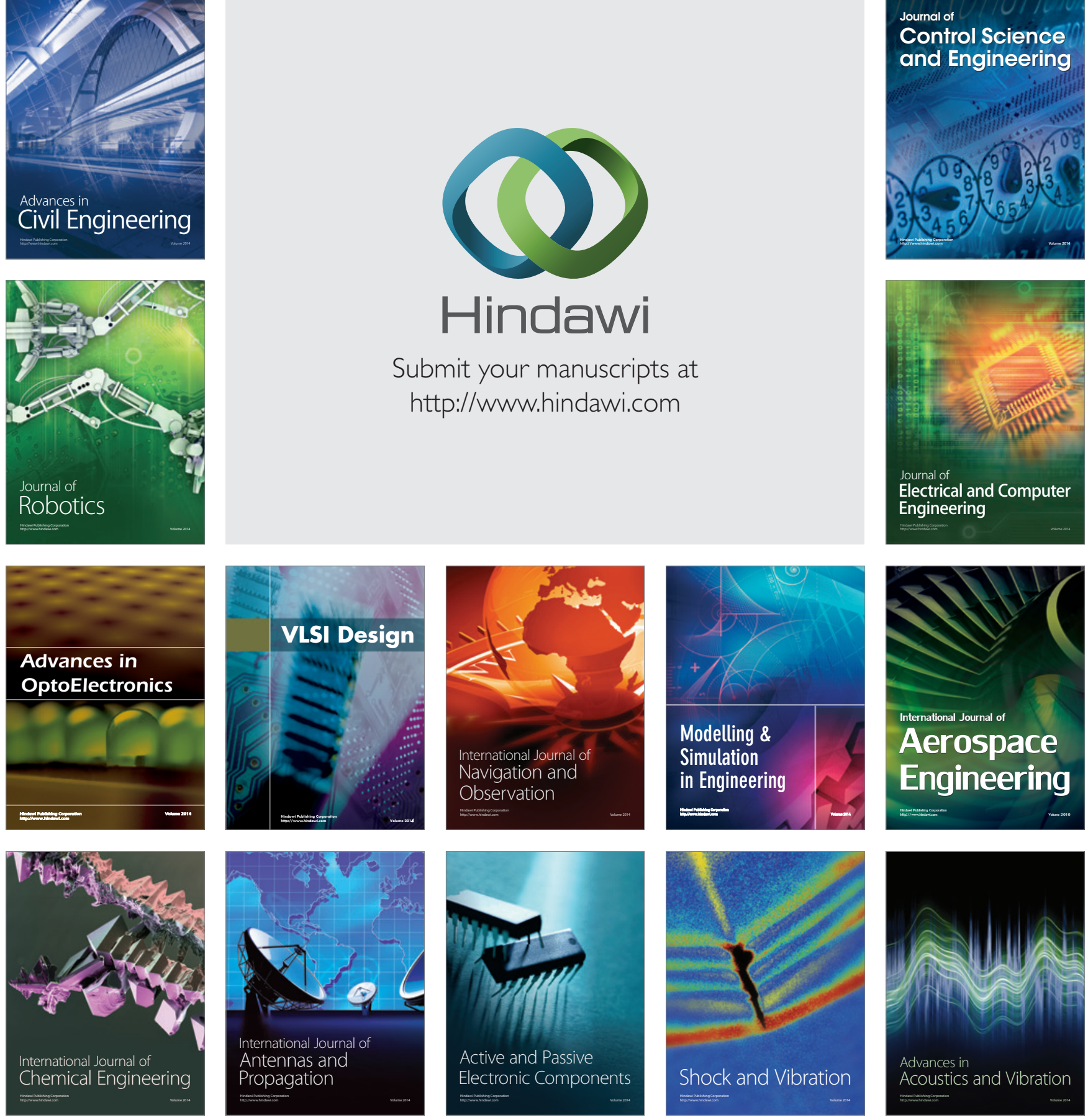